American Journal of Applied Sciences 5 (12): 1693-1700, 2008

ISSN 1546-9239

(C) 2008 Science Publications

\title{
Computational Investigation of Elastoplastic Deformation of a Sandwich Beam on the Elastic Foundation
}

\author{
Moh'd Ishaq Abu Halawa \\ Umar Al Mukhtar University, El-Beida, P.O. Box 919, Libya
}

\begin{abstract}
Elastoplastic bending of a sandwich beam with a rigid compressible filler on an elastic base has been studied. To describe kinematics of asymmetrical across thickness pack in the bearing layers the Bernoulli hypotheses have been accepted. Displacements in the filler vary linearly over thickness. The distributed superficial loading simulates the hydrostatic effect of the ambient liquid. The reaction of the base is described by Winkler's model. A system of equilibrium equations for displacements has been obtained and solved. The numerical results for a sandwich metal-polymer-metal beam are cited.
\end{abstract}

Key words: Computational investigation elasticity, plasticity, sandwich beam, compressible filler, elastic foundation

\section{INTRODUCTION}

The sandwich structures turn to be most rational in conditions of bending strains, i.e. they are most close to optimal ones from the viewpoint of involving minimum weight aspect under given strength and stiffness limits. They have found wide application in intensively developing fields of construction and industry (aviation, rocket production, transport machinery, reactor instrumentation). The static and dynamic strains in the thermal force fields of three-layered elements whose structure is unconjugated to the elastic base have been studied elsewhere ${ }^{[1,2,4,6-9]}$.

The present research sets forth the results of the bending problem of a sandwich beam propping against an elastic base.

Statement and solution of the problem: A three-layer asymmetric in thickness rod with compressible filler is considered. Its external bearing layers are made of elastoplastic material and the filler is of nonlinearly elastic one. To describe kinematics of the pack the following suppositions are accepted: Bernoulli's hypotheses are met in the bearing layers and the exact relations of the theory of elasticity with linear approximation of transitions of its points from coordinate $\mathrm{z}$ are valid in the rigid filler. The conditions of continuity of transitions are used on the contact boundaries. The materials of the bearing layers are incompressible in transversal direction; the contraction and small strains in the filler are considered.

Frame $\mathrm{x}, \mathrm{y}, \mathrm{z}$ is related to the median plane of the filler. Flexures and longitudinal transitions of median surfaces of the bearing layers are designated through $\mathrm{w}_{\mathrm{k}}$

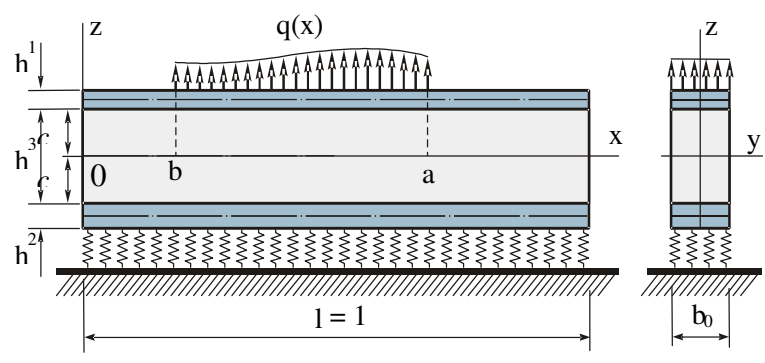

Fig. 1: Analytical model of sandwich beam on an elastic foundation

(x) and $\mathrm{u}_{\mathrm{k}}(\mathrm{x}) ; \mathrm{h}_{\mathrm{k}}$ - thickness the of k-th layer, $\mathrm{h}_{3}=2 \mathrm{c}(\mathrm{k}$ $=1,2,3$ - number of the layer); $b_{0}$ - rod width. The distributed superficial loading $\mathrm{q}(\mathrm{x})$ simulates the hydrostatic effect of the ambient liquid. It is applied to the exterior plane of the first layer (fig. 1). On the lower surface of the second bearing layer the response of the foundation $\mathrm{q}_{\mathrm{R}}(\mathrm{x})$ operates.

Longitudinal and cross transitions in layers $\mathrm{u}^{(\mathrm{k})}(\mathrm{x}$, $\mathrm{z})$ and $\mathrm{w}^{(\mathrm{k})}(\mathrm{x}, \mathrm{z})$ are expressed through four required functions $\mathrm{w}_{1}(\mathrm{x}), \mathrm{u}_{1}(\mathrm{x}), \mathrm{w}_{2}(\mathrm{x})$ and $\mathrm{u}_{2}(\mathrm{x})$ :

$$
\begin{aligned}
& \mathrm{u}^{(1)}=\mathrm{u}_{1}-\left(\mathrm{z}-\mathrm{c}-\frac{\mathrm{h}_{1}}{2}\right) \mathrm{w}_{1},_{\mathrm{x}}, \mathrm{w}^{(1)}=\mathrm{w}_{1}\left(\mathrm{c} \leq \mathrm{z} \leq \mathrm{c}+\mathrm{h}_{1}\right), \\
& \mathrm{u}^{(2)}=\mathrm{u}_{2}-\left(\mathrm{z}+\mathrm{c}+\frac{\mathrm{h}_{2}}{2}\right) \mathrm{w}_{2},{ }_{\mathrm{x}}, \mathrm{w}^{(2)}=\mathrm{w}_{2}\left(-\mathrm{c}-\mathrm{h}_{2} \leq \mathrm{z} \leq-\mathrm{c}\right) ; \\
& \mathrm{u}^{(3)}=\left(1+\frac{\mathrm{z}}{\mathrm{c}}\right)\left(\frac{1}{2} \mathrm{u}_{1}+\frac{\mathrm{h}_{1}}{4} \mathrm{w}_{1},_{\mathrm{x}}\right)+\left(1-\frac{\mathrm{z}}{\mathrm{c}}\right)\left(\frac{1}{2} \mathrm{u}_{2}-\frac{\mathrm{h}_{2}}{4} \mathrm{w}_{2},_{\mathrm{x}}\right) \\
& \mathrm{w}^{(3)}=\frac{1}{2}\left(1+\frac{\mathrm{z}}{\mathrm{c}}\right) \mathrm{w}_{1}+\frac{1}{2}\left(1-\frac{\mathrm{z}}{\mathrm{c}}\right) \mathrm{w}_{2}(-\mathrm{c} \leq \mathrm{z} \leq \mathrm{c})
\end{aligned}
$$


Here, $\mathrm{z}$ - distance from the considered filament to the median plane of the filler; the comma in the superscript index designates operation of derivation on the coordinate following it.

The components of the strain tensor in the layers will be obtained using Cauchy's relations and expression (1):

$$
\begin{aligned}
& \varepsilon_{\mathrm{x}}^{(1)}=\mathrm{u}_{1}, \mathrm{x}_{\mathrm{x}}-\left(\mathrm{z}-\mathrm{c}-\frac{\mathrm{h}_{1}}{2}\right) \mathrm{w}_{1},_{\mathrm{xx}}\left(\mathrm{c} \leq \mathrm{z} \leq \mathrm{c}+\mathrm{h}_{1}\right) ; \\
& \varepsilon_{\mathrm{x}}^{(3)}=\left(1+\frac{\mathrm{z}}{\mathrm{c}}\right)\left(\frac{1}{2} \mathrm{u}_{1},{ }_{\mathrm{x}}+\frac{\mathrm{h}_{1}}{4} \mathrm{w}_{1},{ }_{\mathrm{xx}}\right)+\left(1-\frac{\mathrm{z}}{\mathrm{c}}\right)\left(\frac{1}{2} \mathrm{u}_{2},{ }_{\mathrm{x}}-\frac{\mathrm{h}_{2}}{4} \mathrm{w}_{2},{ }_{\mathrm{xx}}\right), \\
& \varepsilon_{\mathrm{xz}}^{(3)}=\left(\frac{2 \mathrm{z}+\mathrm{h}_{1}}{8 \mathrm{c}}+\frac{1}{4}\right) \mathrm{w}_{1},{ }_{\mathrm{x}}+\left(\frac{-2 \mathrm{z}+\mathrm{h}_{2}}{8 \mathrm{c}}+\frac{1}{4}\right) \mathrm{w}_{2},{ }_{\mathrm{x}}+\frac{\mathrm{u}_{1}-\mathrm{u}_{2}}{4 \mathrm{c}}, \varepsilon_{\mathrm{z}}^{(3)}=\frac{1}{2 \mathrm{c}}\left(\mathrm{w}_{1}-\mathrm{w}_{2}\right),(-\mathrm{c} \leq \mathrm{z} \leq \mathrm{c}) ; \\
& \varepsilon_{\mathrm{x}}^{(2)}=\mathrm{u}_{2},{ }_{\mathrm{x}}-\left(\mathrm{z}+\mathrm{c}+\frac{\mathrm{h}_{2}}{2}\right) \mathrm{w}_{2},{ }_{\mathrm{xx}}\left(-\mathrm{c}-\mathrm{h}_{2} \leq \mathrm{z} \leq-\mathrm{c}\right) ; \varepsilon_{\mathrm{xz}}^{(1)}=\varepsilon_{\mathrm{xz}}^{(2)}=0
\end{aligned}
$$

Spherical and deviator parts of the strain tensor in the considered case will be the following $\left(\ni_{i j}=\varepsilon_{i j}-\varepsilon \delta_{i j} ; \mathrm{i}\right.$, $\mathrm{j}=\mathrm{x}, \mathrm{y}, \mathrm{z})$ :

$$
\varepsilon^{(\mathrm{k})}=\frac{1}{3} \varepsilon_{\mathrm{x}}^{(\mathrm{k})} ; \Im_{\mathrm{x}}^{(\mathrm{k})}=\frac{2}{3} \varepsilon_{\mathrm{x}}^{(\mathrm{k})}(\mathrm{k}=1,2) ; \varepsilon^{(3)}=\frac{1}{3}\left(\varepsilon_{\mathrm{x}}^{(3)}+\varepsilon_{\mathrm{z}}^{(3)}\right) ; \ni_{\mathrm{x}}^{(3)}=\frac{2}{3} \varepsilon_{\mathrm{x}}^{(3)}-\frac{1}{3} \varepsilon_{\mathrm{z}}^{(3)}
$$

Let us introduce the interior strains and moments:

$$
\begin{aligned}
& \mathrm{N}_{\mathrm{x}}^{(\mathrm{k})}=\mathrm{b}_{0} \int_{\mathrm{h}_{\mathrm{k}}} \sigma_{\mathrm{x}}^{(\mathrm{k})} \mathrm{dz} ; \mathrm{M}_{\mathrm{x}}^{(\mathrm{k})}=\mathrm{b}_{0} \int_{\mathrm{h}_{\mathrm{k}}} \sigma_{\mathrm{x}}^{(\mathrm{k})} \mathrm{zdz} ; \\
& \mathrm{Q}^{(3)}=\mathrm{b}_{0} \int_{\mathrm{h}_{3}} \sigma_{\mathrm{xz}}^{(3)} \mathrm{dz} ; \mathrm{N}_{\mathrm{z}}^{(3)}=\mathrm{b}_{0} \int_{\mathrm{h}_{3}} \sigma_{\mathrm{z}}^{(3)} \mathrm{dz} ; \mathbf{M}_{\mathrm{xz}}^{(3)}=\mathrm{b}_{0} \int_{\mathrm{h}_{3}} \sigma_{\mathrm{xz}}^{(3)} \mathrm{zdz}
\end{aligned}
$$

where, $\sigma_{\mathrm{x}}^{(\mathrm{k})} \sigma_{\mathrm{xz}}^{(3)}, \sigma_{\mathrm{z}}^{(3)}$ - components of the strain tensor; integrals are taken over thickness of the k-th layer, $1_{0}$-width of the rod.

Equilibrium Eq. of the considered rod follow a variational principle of Lagrange ${ }^{[3]}$ :

$$
\delta \mathrm{A}_{\mathrm{e}}-\delta \mathrm{A}_{\mathrm{i}}=0,
$$

where, $\delta \mathrm{A}_{\mathrm{e}}$-variation of the work of exterior forces; $\delta \mathrm{A}_{\mathrm{I}}$-variation of the work of interior forces of elasticity;

$$
\begin{aligned}
& \delta A=b_{0} \int_{0}^{1}\left(q \delta w_{1}-q_{R} \delta w_{2}\right) d x \\
& \delta A_{i}=b_{0} \int_{0}^{1}\left[\sum_{k=1}^{3} \int_{h_{k}} \sigma_{x}^{(k)} \delta \varepsilon_{x}^{(k)} d z+2 \int_{h_{3}} \sigma_{x z}^{(3)} \delta \varepsilon_{x z}^{(3)} d z+\int_{h_{3}} \sigma_{z}^{(3)} \delta \varepsilon_{z}^{(3)} d z\right] d x
\end{aligned}
$$

The basic physical Eq. of Ilyushin's theory of small elastoplastic ${ }^{[5]}$ deformations are used for the rod layers. In the deviator-spherical form they are as follows:

$$
s_{\mathrm{ij}}^{(\mathrm{k})}=2 \mathrm{G}_{\mathrm{k}}\left(1-\omega^{(\mathrm{k})}\left(\varepsilon_{\mathrm{u}}^{(\mathrm{k})}\right)\right) \xi_{\mathrm{ij}}^{(\mathrm{k})}, \sigma^{(\mathrm{k})}=3 \mathrm{~K}_{\mathrm{k}} \varepsilon^{(\mathrm{k})},(\mathrm{k}=1,2,3 ; \mathrm{i}, \mathrm{j}=\mathrm{x}, \mathrm{y}, \mathrm{z})
$$

Here $s_{i j}^{(k)}, \sigma^{(k)}$ - deviator and spherical parts of stress tensor; $\vartheta_{i j}^{(k)}, \varepsilon^{(k)}$ - deviator and spherical parts of strain tensor (2); $\varepsilon_{\mathrm{i}}^{(\mathrm{k})}$-deformation intensity in $\mathrm{k}$-th layer,

$$
\varepsilon_{\mathrm{i}}^{(\mathrm{k})}=\frac{\sqrt{2}}{3}\left[\left(\varepsilon_{\mathrm{xx}}^{(\mathrm{k})}-\varepsilon_{\mathrm{yy}}^{(\mathrm{k})}\right)^{2}+\left(\varepsilon_{\mathrm{yy}}^{(\mathrm{k})}-\varepsilon_{\mathrm{zz}}^{(\mathrm{k})}\right)^{2}+\left(\varepsilon_{\mathrm{zz}}^{(\mathrm{k})}-\varepsilon_{\mathrm{xx}}^{(\mathrm{k})}\right)^{2}+6\left(\left(\varepsilon_{\mathrm{xy}}^{(\mathrm{k})}\right)^{2}+\left(\varepsilon_{\mathrm{yz}}^{(\mathrm{k})}\right)^{2}+\left(\varepsilon_{\mathrm{zx}}^{(\mathrm{k})}\right)^{2}\right)\right]^{\frac{1}{2}} ;
$$


$\omega^{(\mathrm{k})}\left(\varepsilon_{\mathrm{i}}^{(\mathrm{k})}\right)$ - at $\mathrm{k}=1,2$ they are the functions of Ilyushin's plasticity, in the case $\varepsilon_{\mathrm{i}}^{(\mathrm{k})} \leq \varepsilon_{\mathrm{y}}^{(\mathrm{k})}$ it is necessary to accept them equal to zero; $\varepsilon_{\mathrm{y}}^{(\mathrm{k})}$ - yield point of the materials of the bearing layers; $\omega^{(3)}\left(\varepsilon_{\mathrm{i}}^{(3)}\right)$ - the universal function describing physical nonlinearity of the filler and $\omega^{(3)} \equiv 0$ at $\varepsilon_{\mathrm{i}}^{(3)} \leq \varepsilon_{\mathrm{s}}^{(3)} ; \varepsilon_{\mathrm{s}}^{(3)}$-physical nonlinearity threshold of the material of the filler; $G_{k}, K_{k}-$ shift and volumetric deformation modules.

Let us consider a method of solving the boundary value problem. Proceeding from relations (7), we shall select the elastic (with index «0») and nonlinear (with index $\langle\omega »)$ members in the stress tensor:

$$
\begin{aligned}
& \sigma_{\mathrm{ij}}^{(\mathrm{k})}=\sigma_{\mathrm{ij}}^{(\mathrm{k}) 0}-\sigma_{\mathrm{ij}}^{(\mathrm{k}) \omega} ; \sigma_{\mathrm{ij}}^{(\mathrm{k}) 0}=2 \mathrm{G}_{\mathrm{k}} \ni_{\mathrm{ij}}^{(\mathrm{k})} \\
& +3 \mathrm{~K}_{\mathrm{k}} \varepsilon^{(\mathrm{k})} \delta_{\mathrm{ij}} ; \sigma_{\mathrm{ij}}^{(\mathrm{k}) \omega}=2 \mathrm{G}_{\mathrm{k}} \omega^{(\mathrm{k})} \ni_{\mathrm{ij}}^{(\mathrm{k})} ;
\end{aligned}
$$

For the considered three-layer rod, in view of formulas for the deviator and the spherical part of the strain tensor (3), we shall have:

in the bearing layers

$$
\begin{aligned}
& \sigma_{x x}^{(\mathrm{k})}=\sigma_{\mathrm{xx}}^{(\mathrm{k}) 0}-\sigma_{\mathrm{xx}}^{(\mathrm{k}) \omega} \\
& \sigma_{\mathrm{xx}}^{(\mathrm{k}) 0}=2 \mathrm{G}_{\mathrm{k}} \exists_{\mathrm{xx}}^{(\mathrm{k})}+3 \mathrm{~K}_{\mathrm{k}} \varepsilon^{(\mathrm{k})}=\mathrm{K}_{\mathrm{k}}^{+} \varepsilon_{\mathrm{xx}}^{(\mathrm{k})} \\
& \sigma_{\mathrm{xx}}^{(\mathrm{k}) \omega}=2 \mathrm{G}_{\mathrm{k}} \exists_{\mathrm{xx}}^{(\mathrm{k})} \omega^{(\mathrm{k})}=\frac{4}{3} \mathrm{G}_{\mathrm{k}} \varepsilon_{\mathrm{xx}}^{(\mathrm{k})} \omega^{(\mathrm{k})}
\end{aligned}
$$

in the filler

$$
\begin{aligned}
& \sigma_{\mathrm{xx}}^{(3)}=\sigma_{\mathrm{xx}}^{(3) 0}-\sigma_{\mathrm{xx}}^{(3) \omega} ; \sigma_{\mathrm{xx}}^{(3) 0}=2 \mathrm{G}_{3} \ni_{\mathrm{xx}}^{(3)}+3 \mathrm{~K}_{3} \varepsilon^{(3)}=\mathrm{K}_{3}^{+} \varepsilon_{\mathrm{xx}}^{(3)}+\mathrm{K}_{3}^{-} \varepsilon_{\mathrm{zz}}^{(3)} ; \\
& \sigma_{\mathrm{xx}}^{(3) \omega}=2 \mathrm{G}_{3} \exists_{\mathrm{xx}}^{(3)} \omega^{(3)}=\frac{2}{3} \mathrm{G}_{3}\left(2 \varepsilon_{\mathrm{xx}}^{(3)}-\varepsilon_{\mathrm{zz}}^{(3)}\right) \omega^{(3)} ; \sigma_{\mathrm{zz}}^{(3)}=\sigma_{\mathrm{zz}}^{(3) 0}-\sigma_{\mathrm{zz}}^{(3) \omega} ; \\
& \sigma_{\mathrm{zz}}^{(3) 0}=2 \mathrm{G}_{3} \exists_{\mathrm{zz}}^{(3)}+3 \mathrm{~K}_{3} \varepsilon^{(3)}=\mathrm{K}_{3}^{+} \varepsilon_{\mathrm{zz}}^{(3)}+\mathrm{K}_{3}^{-} \varepsilon_{\mathrm{xx}}^{(3)} ; \sigma_{\mathrm{zz}}^{(3) \omega}=2 \mathrm{G}_{3} \ni_{\mathrm{zz}}^{(3)} \omega^{(3)}=\frac{2}{3} \mathrm{G}_{3}\left(2 \varepsilon_{\mathrm{zz}}^{(3)}-\varepsilon_{\mathrm{xx}}^{(3)}\right) \omega^{(3)} ; \\
& \sigma_{\mathrm{xz}}^{(3)}=\sigma_{\mathrm{xz}}^{(3) 0}-\sigma_{\mathrm{xz}}^{(3) \omega} ; \sigma_{\mathrm{xz}}^{(3) 0}=2 \mathrm{G}_{3} \ni_{\mathrm{xz}}^{(3)}=2 \mathrm{G}_{3} \varepsilon_{\mathrm{xz}}^{(3)} ; \sigma_{\mathrm{xz}}^{(3) \omega}=2 \mathrm{G}_{3} \exists_{\mathrm{xz}}^{(3)} \omega^{(3)}=2 \mathrm{G}_{3} \varepsilon_{\mathrm{xz}}^{(3)} \omega^{(3)}
\end{aligned}
$$

Here, $\omega^{(\mathrm{k})} \equiv \omega^{(\mathrm{k})}\left(\varepsilon_{\mathrm{i}}^{(\mathrm{k})}\right)$ - universal functions of nonlinearity of materials of the layers defined experimentally; $\mathrm{K}_{\mathrm{k}}^{+}=\mathrm{K}_{\mathrm{k}}+\frac{4}{3} \mathrm{G}_{\mathrm{k}} ; \mathrm{K}_{\mathrm{k}}^{-}=\mathrm{K}_{\mathrm{k}}-\frac{2}{3} \mathrm{G}_{\mathrm{k}}$.

After similar (9) manipulations with interior strains (4), we get:

$$
\begin{aligned}
& \mathrm{N}_{\mathrm{x}}^{(\mathrm{k})}=\mathrm{N}_{\mathrm{x}}^{(\mathrm{k}) 0}-\mathrm{N}_{\mathrm{x}}^{(\mathrm{k}) \omega} ; \mathrm{N}_{\mathrm{z}}^{(3)}=\mathrm{N}_{\mathrm{z}}^{(3) 0}-\mathrm{N}_{\mathrm{z}}^{(3) \omega} ; \\
& \mathrm{M}_{\mathrm{x}}^{(\mathrm{k})}=\mathrm{M}_{\mathrm{x}}^{(\mathrm{k}) 0}-\mathrm{M}_{\mathrm{x}}^{(\mathrm{k}) \omega} ; \mathrm{M}_{\mathrm{xz}}^{(3)}=\mathrm{M}_{\mathrm{xz}}^{(3) 0}-\mathrm{M}_{\mathrm{xz}}^{(3) \omega} ; \\
& \mathrm{Q}^{(3)}=\mathrm{Q}^{(3) 0}-\mathrm{Q}^{(3) \omega}
\end{aligned}
$$

The basic (with index «0») and additional (with index « calculated by the following formulas:

in the bearing layers

$$
\begin{aligned}
& \mathrm{N}_{\mathrm{x}}^{(\mathrm{k}) 0}=\mathrm{b}_{0} \int_{\mathrm{h}_{\mathrm{k}}} \sigma_{\mathrm{xx}}^{(\mathrm{k}) 0} \mathrm{dz}=\mathrm{b}_{0} \int_{\mathrm{h}_{\mathrm{k}}} \mathrm{K}_{\mathrm{k}}^{+} \varepsilon_{\mathrm{xx}}^{(\mathrm{k})} \mathrm{dz} ; \mathrm{N}_{\mathrm{x}}^{(\mathrm{k}) \omega}=\mathrm{b}_{0} \int_{\mathrm{h}_{\mathrm{k}}} \sigma_{\mathrm{xx}}^{(\mathrm{k}) \omega} \mathrm{dz}=\frac{4}{3} \mathrm{~b}_{0} \int_{\mathrm{h}_{\mathrm{k}}} \mathrm{G}_{\mathrm{k}} \varepsilon_{\mathrm{xx}}^{(\mathrm{k})} \omega^{(\mathrm{k})} \mathrm{dz} ; \\
& \mathrm{M}_{\mathrm{x}}^{(\mathrm{k}) 0}=\mathrm{b}_{0} \int_{\mathrm{h}_{\mathrm{k}}} \sigma_{\mathrm{xx}}^{(\mathrm{k}) 0} \mathrm{zdz}=\mathrm{b}_{0} \int_{\mathrm{h}_{\mathrm{k}}} \mathrm{K}_{\mathrm{k}}^{+} \varepsilon_{\mathrm{xx}}^{(\mathrm{k})} \mathrm{zdz} ; \mathrm{M}_{\mathrm{x}}^{(\mathrm{k}) \omega}=\mathrm{b}_{0} \int_{\mathrm{h}_{\mathrm{k}}} \sigma_{\mathrm{xx}}^{(\mathrm{k}) \omega} \mathrm{zdz}=\frac{4}{3} \mathrm{~b}_{0} \int_{\mathrm{h}_{\mathrm{k}}} \mathrm{G}_{\mathrm{k}} \varepsilon_{\mathrm{xx}}^{(\mathrm{k})} \omega^{(\mathrm{k})} \mathrm{zdz} ;
\end{aligned}
$$

in the filler

$$
\begin{aligned}
& \mathrm{N}_{\mathrm{x}}^{(3) 0}=\mathrm{b}_{0} \int_{\mathrm{h}_{3}} \sigma_{\mathrm{xx}}^{(3) 0} \mathrm{dz}=\mathrm{b}_{0} \int_{\mathrm{h}_{3}}\left(\mathrm{~K}_{3}^{+} \varepsilon_{\mathrm{xx}}^{(3)}+\mathrm{K}_{3}^{-} \varepsilon_{\mathrm{zz}}^{(3)}\right) \mathrm{dz} ; \mathrm{N}_{\mathrm{x}}^{(3) \omega}=\mathrm{b}_{0} \int_{\mathrm{h}_{3}} \sigma_{\mathrm{xx}}^{(3) \omega} \mathrm{dz}=\frac{2}{3} \mathrm{~b}_{0} \int_{\mathrm{h}_{3}} \mathrm{G}_{3}\left(2 \varepsilon_{\mathrm{xx}}^{(3)}-\varepsilon_{\mathrm{zz}}^{(3)}\right) \omega^{(3)} \mathrm{dz} ; \\
& \mathrm{N}_{\mathrm{z}}^{(3) 0}=\mathrm{b}_{0} \int_{\mathrm{h}_{3}} \sigma_{\mathrm{zz}}^{(3) 0} \mathrm{dz}=\mathrm{b}_{0} \int_{\mathrm{h}_{3}}\left(\mathrm{~K}_{3}^{+} \varepsilon_{\mathrm{zz}}^{(3)}+\mathrm{K}_{3}^{-} \varepsilon_{\mathrm{xx}}^{(3)}\right) \mathrm{dz} ; \mathrm{N}_{\mathrm{z}}^{(3) \omega}=\mathrm{b}_{0} \int_{\mathrm{h}_{3}} \sigma_{\mathrm{zz}}^{(3) \omega} \mathrm{dz}=\frac{2}{3} \mathrm{~b}_{0} \int_{\mathrm{h}_{3}} \mathrm{G}_{3}\left(2 \varepsilon_{\mathrm{zz}}^{(3)}-\varepsilon_{\mathrm{xx}}^{(3)}\right) \omega^{(3)} \mathrm{dz} ; \\
& \mathrm{M}_{\mathrm{x}}^{(3) 0}=\mathrm{b}_{0} \int_{\mathrm{h}_{3}} \sigma_{\mathrm{xx}}^{(3) 0} \mathrm{zdz}=\mathrm{b}_{0} \int\left(\mathrm{K}_{3}^{+} \varepsilon_{\mathrm{xx}}^{(3)}+\mathrm{K}_{3}^{-} \varepsilon_{\mathrm{zz}}^{(3)}\right) \mathrm{zdz} ; \mathrm{M}_{\mathrm{x}}^{(3) \omega}=\frac{2}{3} \mathrm{~b}_{0} \int_{\mathrm{h}_{3}} \mathrm{G}_{3}\left(2 \varepsilon_{\mathrm{xx}}^{(3)}-\varepsilon_{\mathrm{zz}}^{(3)}\right) \omega^{(3)} \mathrm{zdz} ; \\
& \mathrm{M}_{\mathrm{z}}^{(3) 0}=\mathrm{b}_{0} \int_{\mathrm{h}_{3}} \sigma_{\mathrm{zz}}^{(3) 0} \mathrm{zdz}=\mathrm{b}_{0} \int_{\mathrm{h}_{3}}\left(\mathrm{~K}_{3}^{+} \varepsilon_{\mathrm{zz}}^{(3)}+\mathrm{K}_{3}^{-} \varepsilon_{\mathrm{xx}}^{(3)}\right) \mathrm{zdz} ; \mathrm{M}_{\mathrm{z}}^{(3) \omega}=\frac{2}{3} \mathrm{~b}_{0} \int_{\mathrm{h}_{3}} \mathrm{G}_{3}\left(2 \varepsilon_{\mathrm{zz}}^{(3)}-\varepsilon_{\mathrm{xx}}^{(3)}\right) \omega^{(3)} \mathrm{zdz} ; \\
& \mathrm{Q}^{(3) 0}=\mathrm{b}_{0} \int_{\mathrm{h}_{3}} \sigma_{\mathrm{xz}}^{(3) 0} \mathrm{dz}=2 \mathrm{~b}_{0} \int_{\mathrm{h}_{3}} \mathrm{G}_{3} \varepsilon_{\mathrm{xz}}^{(3) 0} \mathrm{dz} ; \mathrm{Q}^{(3) \omega}=\mathrm{b}_{0} \int_{\mathrm{h}_{3}} \sigma_{\mathrm{xz}}^{(3) \omega} \mathrm{dzb}=2 \mathrm{~b}_{0} \int_{\mathrm{h}_{3}} \mathrm{G}_{3} \varepsilon_{\mathrm{xz}}^{(3)} \omega^{(3)} \mathrm{dz}
\end{aligned}
$$


To correlate the response and the flexure, a well-known Winkler's model is used, according to which

$$
\mathrm{q}_{\mathrm{R}}=\mathrm{\kappa}_{0} \mathrm{w}_{2}
$$

where, $\kappa_{0}$-stiffness coefficient of the elastic foundation and the response of the foundation is always directed to the side opposite to the flexure.

After substitution in (5) the values of (6), using expressions (8)-(11), we shall have a system of four nonlinear differential Eq. in an iterative aspect

$$
\begin{aligned}
& \mathrm{a}_{1} \mathrm{u}_{1}^{\mathrm{n}}-\mathrm{a}_{1} \mathrm{u}_{2}^{\mathrm{n}}-\mathrm{a}_{4} \mathrm{u}_{1}^{\mathrm{n}},_{x x}-\mathrm{a}_{5} \mathrm{u}_{2}^{\mathrm{n}},{ }_{x x}+\mathrm{a}_{2} \mathrm{w}_{1}^{\mathrm{n}},{ }_{x}+\mathrm{a}_{3} \mathrm{w}_{2}^{\mathrm{n}},{ }_{\mathrm{x}}-2 \mathrm{a}_{6} \mathrm{w}_{1}^{\mathrm{n}},_{x x x}+\mathrm{a}_{7} \mathrm{w}_{2}^{\mathrm{n}},{ }_{x x x}=\mathrm{p}_{\omega}^{\mathrm{n}-1} \\
& -\mathrm{a}_{1} \mathrm{u}_{1}^{\mathrm{n}}+\mathrm{a}_{1} \mathrm{u}_{2}^{\mathrm{n}}-\mathrm{a}_{5} \mathrm{u}_{1}^{\mathrm{n}},_{x x}-\mathrm{a}_{9} \mathrm{u}_{2}^{\mathrm{n}},_{x x}-\mathrm{a}_{10} \mathrm{w}_{1}^{\mathrm{n}},_{x}-\mathrm{a}_{17} \mathrm{w}_{2}^{\mathrm{n}},_{x}-\mathrm{a}_{6} \mathrm{w}_{1}^{\mathrm{n}}{ }_{x x x}+2 \mathrm{a}_{7} \mathrm{w}_{2}^{\mathrm{n}},_{x x x}=\mathrm{h}_{\omega}^{\mathrm{n}-1} ;
\end{aligned}
$$

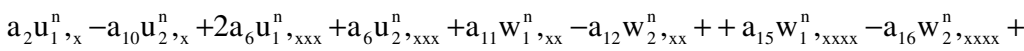

$$
\begin{aligned}
& +\mathrm{a}_{8} \mathrm{w}_{1}^{\mathrm{n}}-\mathrm{a}_{8} \mathrm{w}_{2}^{\mathrm{n}}=\mathrm{q}+\frac{1}{2} \mathrm{p}, \mathrm{h}_{1}+\mathrm{q}_{\omega}^{\mathrm{n}-1}
\end{aligned}
$$

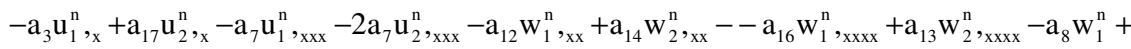

$$
\begin{aligned}
& +\left(\mathrm{a}_{8}+\kappa_{0}\right) \mathrm{w}_{2}^{\mathrm{n}}=\mathrm{g}_{\omega}^{\mathrm{n}-1}
\end{aligned}
$$

where $a_{i}$. $(i=1, \ldots, 17)$-the factors expressed through volumetric and shift module of elasticity of the materials $\mathrm{K}_{\mathrm{k}}, \mathrm{G}_{\mathrm{k}}$ and geometrical parameters of the rod layers;

$$
\begin{aligned}
& \mathrm{p}_{\omega}^{\mathrm{n}-1}=\frac{1}{\mathrm{~b}_{0}}\left(\mathrm{H}_{1}^{\omega(\mathrm{n}-1)}-\mathrm{P}_{1}^{\omega(\mathrm{n}-1)},{ }_{\mathrm{x}}\right) ; \quad \mathrm{q}_{\omega}^{\mathrm{n}-1}=\frac{1}{\mathrm{~b}_{0}}\left(\mathrm{~S}_{1}^{\omega(\mathrm{n}-1)},{ }_{\mathrm{xx}}+\mathrm{H}_{2}^{\omega(\mathrm{n}-1)}-\mathrm{T}_{1}^{\omega(\mathrm{n}-1)},{ }_{\mathrm{x}}\right) ; \\
& \mathrm{g}_{\omega}^{\mathrm{n}-1}=\frac{1}{\mathrm{~b}_{0}}\left(\mathrm{~S}_{2}^{\omega(\mathrm{n} n-1)}{ }_{\mathrm{xx}}-\mathrm{H}_{2}^{\omega(\mathrm{n}-1)}-\mathrm{T}_{2}^{\omega(\mathrm{n}-1)}{ }_{\mathrm{x}}\right) ; \mathrm{H}_{1}^{\omega(\mathrm{n}-1)}=\frac{2 \mathrm{Q}^{(3) \omega(\mathrm{n}-1)}}{\mathrm{c}} ; \mathrm{H}_{2}^{\omega(\mathrm{n}-1)}=\frac{\mathrm{N}_{\mathrm{z}}^{(3) \omega(\mathrm{n}-1)}}{2 \mathrm{c}} ; \\
& \mathrm{T}_{1}^{\omega(\mathrm{n} n-1)}=\left(1+\frac{\mathrm{h}_{1}}{2 \mathrm{c}}\right) \frac{\mathrm{Q}^{(3) \omega(\mathrm{n}-1)}}{2}+\frac{\mathrm{M}_{\mathrm{xz}}^{(3) \omega(\mathrm{n}-1)}}{2 \mathrm{c}} ; \mathrm{T}_{2}^{(\omega(\mathrm{n}-1)}=\left(1+\frac{\mathrm{h}_{2}}{2 \mathrm{c}}\right) \frac{\mathrm{Q}^{(3) \omega(\mathrm{n}-1)}}{2}-\frac{\mathrm{M}_{\mathrm{xz}}^{(3) \omega(\mathrm{n}-1)}}{2 \mathrm{c}} ; \\
& S_{1}^{\omega(n-1)}=\left(c+\frac{h_{1}}{2}\right) N_{x}^{(1) \omega(n-1)}-M_{x}^{(1) \omega(n-1)}+\frac{h_{1}}{4} N_{x}^{(3) \omega(n-1)}+\frac{h_{1}}{4 c} M_{x}^{(3) \omega(n-1)} ; \\
& S_{2}^{\omega(n-1)}=-\left(c+\frac{h_{2}}{2}\right) N_{x}^{(2) \omega(n-1)}-M_{x}^{(2) \omega(n-1)}-\frac{h_{2}}{4} N_{x}^{(3) \omega(n-1)}+\frac{h_{2}}{4 c} M_{x}^{(3) \omega(n-1)} ; \\
& \mathrm{P}_{1}^{\omega(\mathrm{n}-1)}=\frac{N_{\mathrm{x}}^{(3) \omega(\mathrm{n}-1)}}{2}+\frac{M_{\mathrm{x}}^{(3) \omega(\mathrm{n}-1)}}{2 \mathrm{c}}+\mathrm{N}_{\mathrm{x}}^{(1) \omega(\mathrm{n}-1)} ; \mathrm{P}_{2}^{\omega(\mathrm{n}-1)}=\frac{N_{\mathrm{x}}^{(3) \omega(\mathrm{n}-1)}}{2}-\frac{M_{\mathrm{x}}^{(3) \omega(\mathrm{n}-1)}}{2 \mathrm{c}}+\mathrm{N}_{\mathrm{x}}^{(2) \omega(\mathrm{n}-1)} ; \\
& \mathrm{N}_{\mathrm{x}}^{(\mathrm{k}) \omega(\mathrm{n}-1)}=\frac{4}{3} \mathrm{~b}_{0} \int_{\mathrm{h}_{\mathrm{k}}} \mathrm{G}_{\mathrm{k}} \varepsilon_{\mathrm{x}}^{(\mathrm{k})(\mathrm{n}-1)} \omega^{(\mathrm{k})}\left(\varepsilon_{\mathrm{u}}^{(\mathrm{k})(\mathrm{n}-1)}\right) \mathrm{dz}(\mathrm{k}=1,2) ; \\
& \mathbf{M}_{\mathrm{x}}^{(\mathrm{k}) \omega(\mathrm{n}-1)}=\frac{4}{3} \mathrm{~b}_{0} \int_{\mathrm{h}_{\mathrm{k}}} \mathrm{G}_{\mathrm{k}} \varepsilon_{\mathrm{x}}^{(\mathrm{k})(\mathrm{n}-1)} \omega^{(\mathrm{k})}\left(\varepsilon_{\mathrm{u}}^{(\mathrm{k})(\mathrm{n}-1)}\right) \mathrm{zdz}(\mathrm{k}=1,2) ; \\
& N_{\mathrm{x}}^{(3) \omega(\mathrm{n}-1)}=\frac{2}{3} \mathrm{~b}_{0} \int_{\mathrm{h}_{3}}\left[\mathrm{G}_{3}\left(2 \varepsilon_{\mathrm{x}}^{(3)(\mathrm{n}-1)}-\varepsilon_{\mathrm{z}}^{(3)(\mathrm{n}-1)}\right) \times \omega^{(3)}\left(\varepsilon_{\mathrm{u}}^{(3)(\mathrm{n}-1)}\right)\right] \mathrm{dz} ; \\
& N_{z}^{(3) \omega(n-1)}=\frac{2}{3} b_{0} \int\left[G_{3}\left(2 \varepsilon_{z}^{(3)(n-1)}-\varepsilon_{x}^{(3)(n-1)}\right) \times \omega^{(3)}\left(\varepsilon_{u}^{(3)(n-1)}\right)\right] d z ; \\
& M_{\mathrm{x}}^{(3) \omega(\mathrm{n}-1)}=\frac{2}{3} \mathrm{~b}_{0} \int_{\mathrm{h}_{3}}\left[\mathrm{G}_{3}\left(2 \varepsilon_{\mathrm{x}}^{(3)(\mathrm{n}-1)}-\varepsilon_{\mathrm{z}}^{(3)(\mathrm{n}-1)}\right) \times \omega^{(3)}\left(\varepsilon_{\mathrm{u}}^{(3)(\mathrm{n}-1)}\right)\right] \mathrm{zdz} ; \\
& M_{z}^{(3) \omega(n-1)}=\frac{2}{3} b_{0} \int_{h_{3}}\left[G_{3}\left(2 \varepsilon_{z}^{(3)(n-1)}-\varepsilon_{x}^{(3)(n-1)}\right) \times \omega^{(3)}\left(\varepsilon_{u}^{(3)(n-1)}\right)\right] z d z ; \\
& \mathrm{Q}^{(3) \omega(\mathrm{n}-1)}=2 \mathrm{~b}_{0} \int_{\mathrm{h}_{3}} \mathrm{G}_{3} \varepsilon_{\mathrm{xz}}^{(3)(\mathrm{n}-1)} \omega^{(3)}\left(\varepsilon_{\mathrm{u}}^{(3)(\mathrm{n}-1)}\right) \mathrm{dz} .
\end{aligned}
$$

Let us accept the condition of a simply supported rod on the end faces on motionless in space rigid supports. The boundary conditions in sections $\mathrm{x}=0$; 1 (1-rod length) in transitions take the following form: 


$$
\mathrm{w}^{\mathrm{kn}}=\mathrm{u}^{\mathrm{kn}}{ }_{\mathrm{x}}=\mathrm{w}^{\mathrm{kn}}{ }_{{ }_{\mathrm{xx}}}=0(\mathrm{k}=1,2)
$$

where, k-number of the bearing layer, n-number of linear approximation.

Let us assume the solution of a system of differential Eq. (12) in the form of expansions in trigonometric series, which automatically satisfy boundary conditions of resting on a rigid support (13):

$$
\begin{aligned}
& \mathrm{u}_{1}^{\mathrm{n}}=\sum_{\mathrm{m}=1}^{\infty} \mathrm{U}_{1 \mathrm{~m}}^{\mathrm{n}} \cos \left(\frac{\pi \mathrm{mx}}{1}\right) ; \mathrm{u}_{2}^{\mathrm{n}}=\sum_{\mathrm{m}=1}^{\infty} \mathrm{U}_{2 \mathrm{~m}}^{\mathrm{n}} \cos \left(\frac{\pi \mathrm{mx}}{1}\right) ; \\
& \mathrm{w}_{1}^{\mathrm{n}}=\sum_{\mathrm{m}=1}^{\infty} \mathrm{W}_{1 \mathrm{~m}}^{\mathrm{n}} \sin \left(\frac{\pi \mathrm{mx}}{1}\right) ; \mathrm{w}_{2}^{\mathrm{n}}=\sum_{\mathrm{m}=1}^{\infty} \mathrm{W}_{2 \mathrm{~m}}^{\mathrm{n}} \sin \left(\frac{\pi \mathrm{mx}}{1}\right) ;
\end{aligned}
$$

where $\mathrm{U}_{1 \mathrm{~m}}^{\mathrm{n}}, \mathrm{U}_{2 \mathrm{~m}}^{\mathrm{n}}, \mathrm{W}_{1 \mathrm{~m}}^{\mathrm{n}}, \mathrm{W}_{2 \mathrm{~m}}^{\mathrm{n}}$ - unknown amplitudes of displacements on the $\mathrm{n}$-th step.

Let us present transversal loading $\mathrm{q}(\mathrm{x})$ and additional «exterior» strains in the form of expansions in the following series:

$$
\begin{aligned}
& \mathrm{q}=\sum_{\mathrm{m}=1}^{\infty} \mathrm{q}_{\mathrm{m}} \sin \left(\frac{\pi \mathrm{mx}}{1}\right), \mathrm{q}_{\mathrm{m}}=\frac{2}{1} \int_{0}^{1} \mathrm{q}(\mathrm{x}) \sin \left(\frac{\pi \mathrm{mx}}{1}\right) \mathrm{dx} ; \\
& \mathrm{q}_{\omega}^{\mathrm{n}-1}=\sum_{\mathrm{m}=1}^{\infty} \mathrm{q}_{\omega \mathrm{m}}^{\mathrm{n}-1} \sin \left(\frac{\pi \mathrm{mx}}{1}\right), \mathrm{q}_{\omega \mathrm{m}}^{\mathrm{n}-1}=\frac{2}{1} \int_{0}^{1} \mathrm{q}_{\omega}^{\mathrm{n}-1}(\mathrm{x}) \sin \left(\frac{\pi \mathrm{mx}}{1}\right) \mathrm{dx} ; \\
& \mathrm{g}_{\omega}^{\mathrm{n}-1}=\sum_{\mathrm{m}=1}^{\infty} \mathrm{g}_{\omega \mathrm{m}}^{\mathrm{n}-1} \sin \left(\frac{\pi \mathrm{mx}}{1}\right), \mathrm{g}_{\omega \mathrm{m}}^{\mathrm{n}-1}=\frac{2}{1} \int_{0}^{1} \mathrm{~g}_{\omega}^{\mathrm{n}-1}(\mathrm{x}) \sin \left(\frac{\pi \mathrm{mx}}{1}\right) \mathrm{dx} ; \\
& \mathrm{h}_{\omega}^{\mathrm{n}-1}=\sum_{\mathrm{m}=1}^{\infty} \mathrm{h}_{\omega \mathrm{m}}^{\mathrm{n}-1} \cos \left(\frac{\pi \mathrm{mx}}{1}\right), \mathrm{h}_{\omega \mathrm{m}}^{\mathrm{n}-1}=\frac{2}{1} \int_{0}^{1} \mathrm{~h}_{\omega}^{\mathrm{n}-1}(\mathrm{x}) \cos \left(\frac{\pi \mathrm{mx}}{1}\right) \mathrm{dx} \\
& \mathrm{p}_{\omega}^{\mathrm{n}-1}=\sum_{\mathrm{m}=1}^{\infty} \mathrm{p}_{\omega \mathrm{m}}^{\mathrm{n}-1} \cos \left(\frac{\pi \mathrm{mx}}{1}\right), \mathrm{p}_{\omega \mathrm{m}}^{\mathrm{n}-1}=\frac{2}{1} \int_{0}^{1} \mathrm{p}_{\omega}^{\mathrm{n}-1}(\mathrm{x}) \cos \left(\frac{\pi \mathrm{mx}}{1}\right) \mathrm{dx} .
\end{aligned}
$$

After substitution of displacements (14) and loadings (15) in (12) we receive the following system of linear algebraic Eq.s for required amplitudes of transitions $\mathrm{U}_{1 \mathrm{~m}}^{\mathrm{n}}, \mathrm{U}_{2 \mathrm{~m}}^{\mathrm{n}}, \mathrm{W}_{2 \mathrm{~m}}^{\mathrm{n}}$ :

$$
\left\{\begin{array}{l}
b_{1} U_{1 m}^{n}+b_{2} U_{2 m}^{n}+b_{3} W_{1 m}^{n}+b_{4} W_{2 m}^{n}=p_{\omega m}^{n-1}, \\
b_{2} U_{1 m}^{n}+b_{5} U_{2 m}^{n}+b_{6} W_{1 m}^{n}-b_{7} W_{2 m}^{n}=h_{\omega m}^{n-1}, \\
b_{3} U_{1 m}^{n}+b_{6} U_{2 m}^{n}+b_{8} W_{1 m}^{n}+b_{9} W_{2 m}^{n}=q_{m}+q_{\omega m}^{n-1}, \\
b_{4} U_{1 m}^{n}-b_{7} U_{2 m}^{n}+b_{9} W_{1 m}^{n}+b_{10} W_{2 m}^{n}=g_{\omega m}^{n-1} .
\end{array}\right.
$$

Here, parameters $b_{i}$ are expressed through factors and depend on parameter $\mathrm{m}$.

We shall obtain amplitudes $\mathrm{U}_{1 \mathrm{~m}}, \mathrm{U}_{2 \mathrm{~m}}, \mathrm{~W}_{1 \mathrm{~m}}, \mathrm{~W}_{2 \mathrm{~m}}$ by having solved system (16). Further, by formulas (14) the required functions are calculated. Transitions in the bearing layers and the filler are found by relations (1), deformations by Eq. (2) and strains by Eq. (7).

\section{RESULTS AND DISCUSSION}

The present research of deformation was conducted for a three-layer rod formed of materials D16T - Teflon - D16T. All transitions and linear sizes of the rod are referred to its length 1 . The rated stiffness factors correspond to weak $\left(\kappa_{0}=1\right)$, on the average $\left(\kappa_{0}=100\right)$ and rather rigid $\left(\kappa_{0}=10^{5} \mathrm{MPa} / \mathrm{m}\right)$ foundations. Geometrical parameters of the rod layers: $h_{1}=0,04, h_{2}$ $=0,02, h_{3}=0,18,1=1$. Loading is regularly distributed along the whole length of the rod and presses it into the elastic foundation. Its magnitude was selected according to a rigidity of the foundation so that nonlinear properties of materials of the layers were exhibited sufficiently, but deformations remained small, i.e. within the limits of the accepted model.

Figure $2 \mathrm{a}$ and $\mathrm{b}$ illustrates the process of convergence of the method of elastic solutions at bending of elastoplastic three-layer rod on the foundation with $\kappa_{0}=1 \mathrm{MPa} / \mathrm{m}$ at $\mathrm{q}=-6 \mathrm{MPa}$. The number of the curve in the figures corresponds to the number of iteration. The first approximation is the solution of the problem of the theory of elasticity; the second approximation differs from it on the average by $11 \%$. At each subsequent iteration this difference decreases and the 7-th approximation, which is accepted for a required solution, differs from the 6-th one by less than $1 \%$.
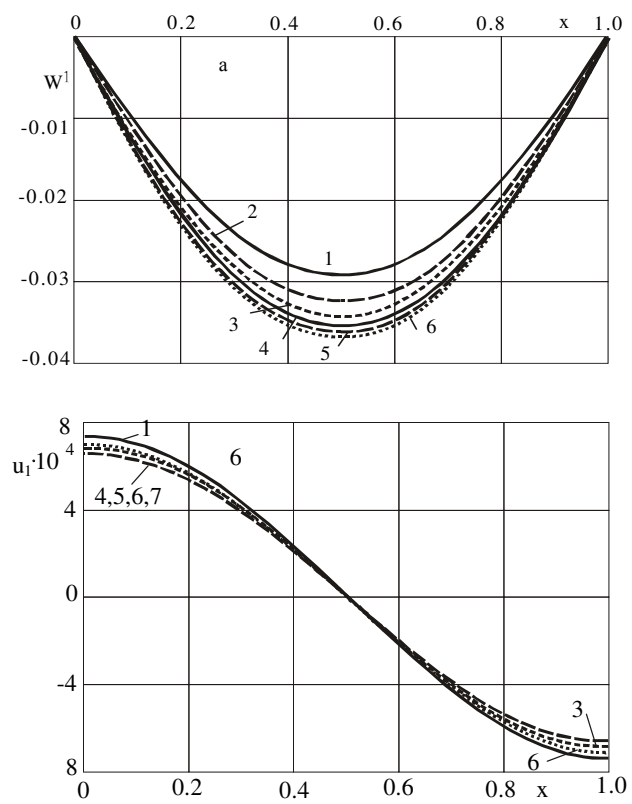

Fig. 2: Process of convergence of the method of elastic solution for elastoplastic sandwich beam on the foundation of small rigidity 

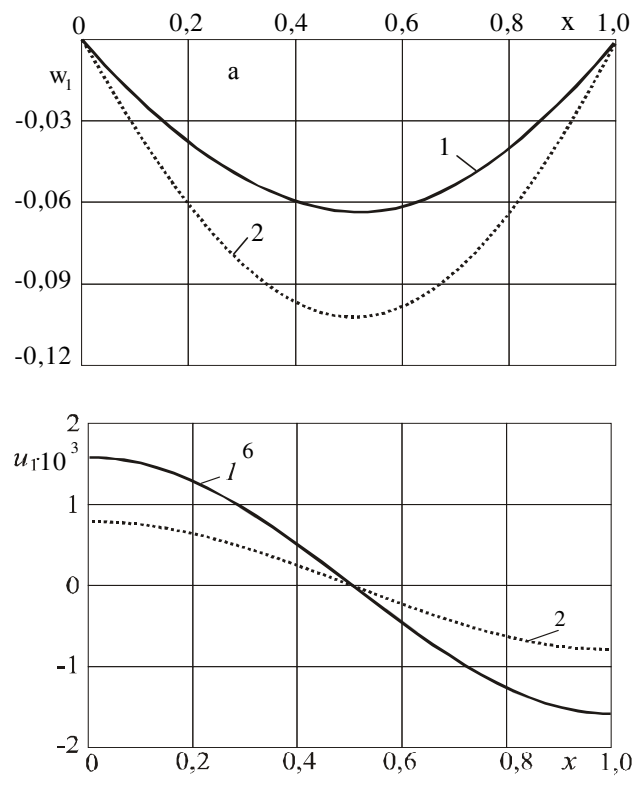

Fig. 3: Influence of physical nonlinearity of materials on flexures $\mathrm{w}_{1}$ and longitudinal displacements $\mathrm{u}_{1}$ in the first layer (foundation of average rigidity)

Further check of convergence of the method has shown a steady approach to zero of the difference between the subsequent and previous approximations.

As a result, elastoplastic flexures of the bearing layers of the rod are approximately by $27 \%$ larger, than elastic ones, longitudinal transitions thus becoming less by $25 \%$.

It is possible to judge about the influence of plasticity and physical nonlinearity of materials upon rated flexures $\mathrm{w} 1$ and longitudinal transitions $\mathrm{u} 1$ in the first layer with the help of Fig. 3 a and b: 1-elastic, 2 elastoplastic $\operatorname{rod}\left(\kappa_{0}=100 \mathrm{MPa} / \mathrm{m}\right)$. At elastoplastic deformation the flexure increases by $59 \%$ in comparison to the elastic, longitudinal displacements thus becoming twice as less. The magnitude of loading is $\mathrm{q}=-18 \mathrm{MPa}$.

In Fig. 4, the areas of nonlinear deformation in the rod layers (dark filling) on the foundation with average rigidity are shown. The filler is deformed till $83 \%$ nonlinearly physically. The areas of plasticity in the bearing layers occupy on the average $35 \%$ of the material volume.

Figure 5 illustrates the regions of nonlinear deformation in the layers of the rod (dark filling) propping against a highly rigid base. The whole filler undergoes physically nonlinear deformation. The regions of plasticity in the bearing layers occupy on the average $90 \%$ of the material volume.

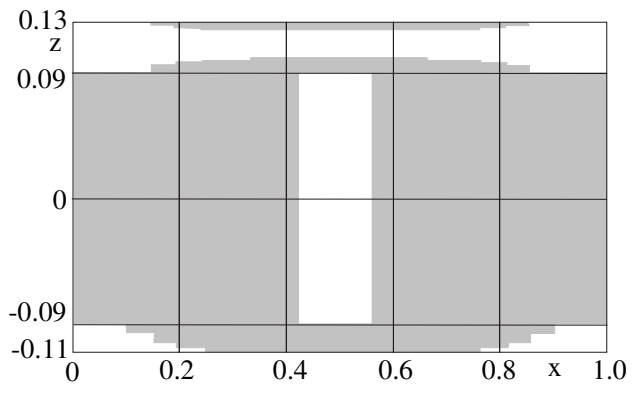

Fig. 4: Zones of plasticity and physical nonlinearity of materials of layers (dark filling) on the foundation with average rigidity

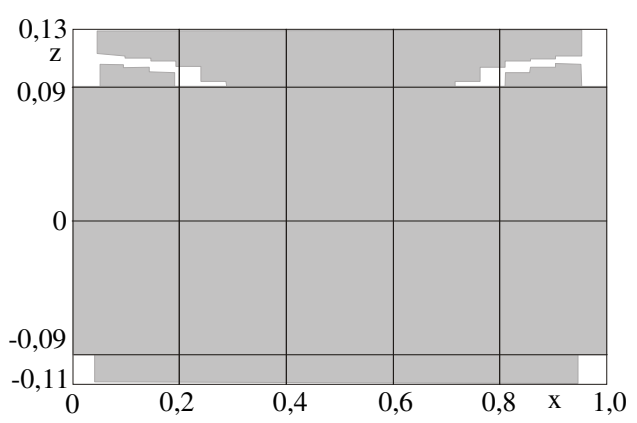

Fig. 5: Zones of plasticity and physical nonlinearity of materials of the layers (dark filling) on a highly rigid base

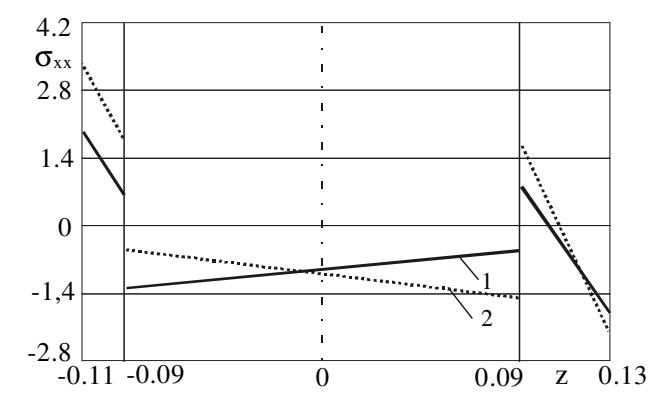

Fig. 6: The distribution of the normal longitudinal stresses $\sigma_{\mathrm{xx}}$ in the median cross-section $(\mathrm{x}=0.5)$

Let us consider the effect of plasticity and physical nonlinearity of the layers upon the stress state in the rod by taking a base of a mean rigidity with the modulus of subgrade reaction $\mathrm{k}_{0}=100 \mathrm{MPa} / \mathrm{m}$. Here and further, stress values in the external layers are related as $\mathrm{q}_{1}=$ $10^{9}$ and in the filler as $\mathrm{q}_{3}=10^{7} \mathrm{~Pa}$. The distribution of the normal longitudinal stresses $\sigma_{\mathrm{xx}}$ in the median cross-section $(x=0.5)$ is shown in Fig. 6, where 1 elastic, 2-elastoplastic rods. The value of normal 


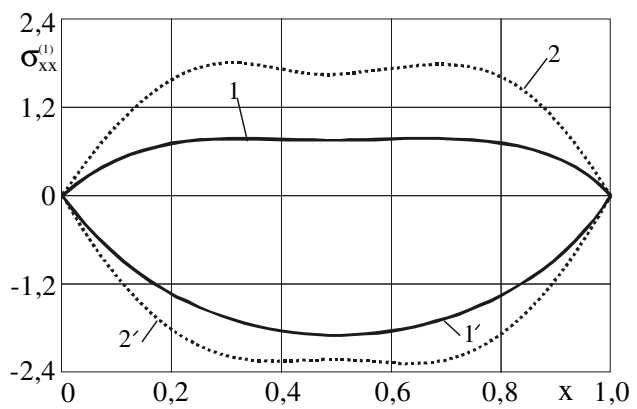

Fig. 7: The distribution of normal stresses over the boundary planes of the first layer

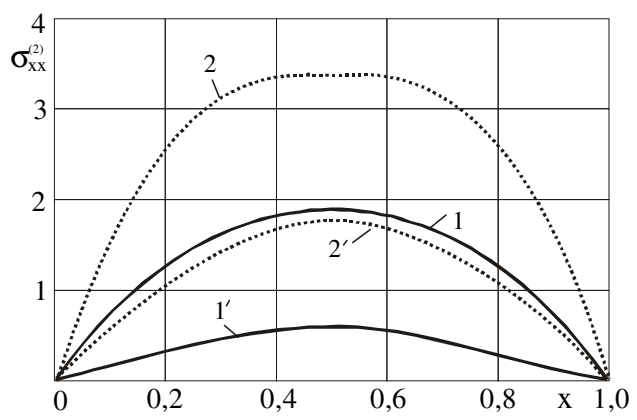

Fig. 8: The distribution of normal stresses over the boundary planes of the second layer

stresses in the bearing layers increases in modules by 100 and $37 \%$, respectively, when plasticity and nonlinearity of materials of the layers are taken into account. The maximal stresses in the filler vary but slightly and remain negative.

The distribution of normal stresses over the boundary planes of the first layer is illustrated in Fig. 7 (stroked $\mathrm{z}=\mathrm{c}+\mathrm{h}_{1}$, without a stroke $\mathrm{z}=\mathrm{c}$ ): 1 - elastic, 2 - elastoplastic rods. The stresses increase by approximately $110 \%$ if the plastic and physically nonlinear properties of materials of the layers over the external plane are taken into account. They are varying similarly though to a lesser degree in the splice with a filler - roughly by $30 \%$.

Figure 8 shows distribution of stresses $\sigma_{x x}^{(2)}$ over the boundary planes of the second bearing layer (primed $\mathrm{z}=-\mathrm{c}$, unprimed $\mathrm{z}=-\mathrm{c}-\mathrm{h}_{2}$ ). Numbering of the curves is the former. The image on the external surface of the layer is like the previous one, where the stresses augment by $90 \%$. In the splice with the filler, the stresses increase by a factor of three.

Changes in the longitudinal $\sigma_{\mathrm{xx}}^{(3)}$ and transversal $\sigma_{z z}^{(3)}$ stresses in the filler along the axis of the rod over the splice planes with the bearing layers is presented in
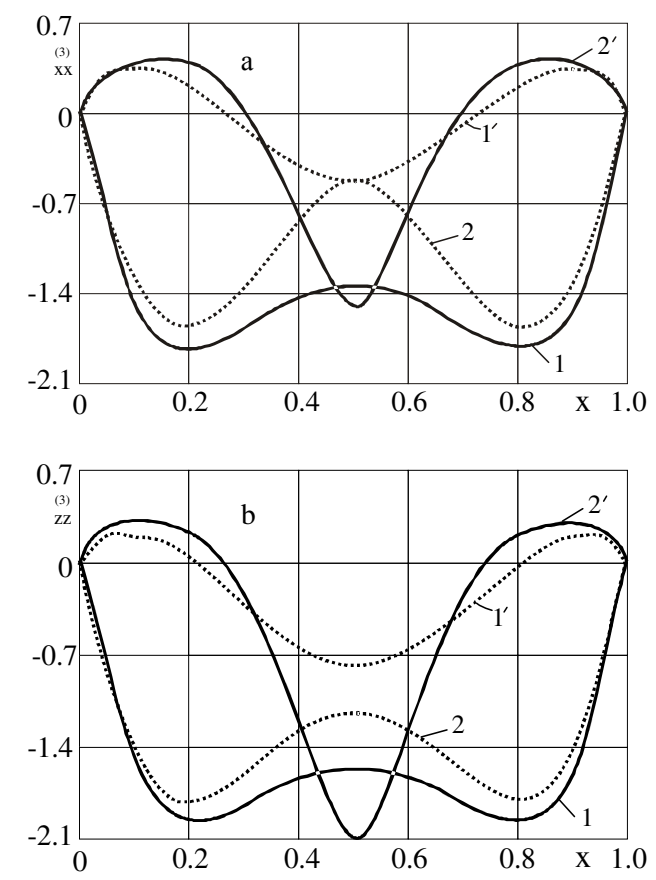

Fig. 9: Variations of longitudinal $\sigma_{x x}^{(3)}$ and transversal $\sigma_{z z}^{(3)}$ stresses in filler along axis of the rod

Fig. 9: $\mathrm{a}$ and $\mathrm{b}$ (unprimed $\mathrm{z}=-\mathrm{c}$, primed $\mathrm{z}=\mathrm{c}$ ): 1 elastic, 2 - elastoplastic rods. The character of the changes in stresses remains intact on both planes when the plastic and physically nonlinear properties of the layer materials are taken into account. The local extremes in the median cross-section of the rod intensify. The stresses on the upper splice near the supports are positive, while in the central part they are negative. In the lower splice, the stresses are of a similar sign.

Consequently, the allowance for the plastic and physically nonlinear properties of materials of the layers in the mechanical-mathematical model renders more precise to the stress-strain state of the sandwich rod with compressible filler resting on an elastic base.

\section{CONCLUSIONS}

Thus, the problem on bending of a sandwich beam resting on an elastic foundation has been stated and solved. As an example, the influence of plastic properties of materials of the layers upon and stress strain state of the beam has been for the foundation of average rigidity. Convergence of the method of elastic solutions has been investigated. 


\section{REFERENCES}

1. Cheng, Zhenqiang, A.K. Jemah and Williams, F.W., 1996. Theory for multilayered anisotropic plates with weakened interfaces. Trans. ASME. J. Appl. Mech., 63 (4): 1019-1026.

2. Ebsioglu, J.K., I966. On the theory on sandwich panels in the reference state. Int. J. Eng. Sci. 6: 166-194.

3. Gorshkov, A.G., E.I. Starovoitov and D.V. Tarlakovskij, 2002. The Theory of Elasticity and Plasticity. FIZMATLIT. Moscow. pp: 416, (Russian).

4. Gorshkov, A.G., E.I. Starovoitov and A.V. Yarovaya, 2005. Mechanics of Laminated Viscous Elastoplastic Constructional Elements. FIZMATLIT. Moscow. pp: 576, (Russian).
5. Ilyushin, A.A., 1948. Plasticity. P.1. Elastoplastic deformation. Gostehizdat. Moscow. pp: 376, (Russian).

6. Starovoitov, E.I., 2002. Viscous elastoplastic laminated plates and shells. BelGUT. Gomel. pp: 343, (Russian).

7. Starovoitov, É.I., D.V. Leonenko and A.V. Yarovaya, 2005. Vibrations of a sandwich rod under local and impulsive forces. Int. Applied Mech. 41 (7): 809-816.

8. Starovoitov, É.I., D.V. Leonenko and A.V. Yarovaya, 2006. Dynamics of three-layer bars. Strength Mater. 41 (7): 668-679.

9. Starovoitov, É.I., A.V. Yarovaya and D.V. Leonenko, 2003. Vibrations of round three-layer plates under the action of various types of surface loads. Strength Mater. 35 (4): 346-352. 